\title{
Radiation induced breast cancer risk in BRCA mutation carriers from low-dose radiological exposures: a systematic review
}

\author{
C. Colin ${ }^{1,2, *}$, N. Foray ${ }^{2}$, G. Di Leo ${ }^{3}$ and F. Sardanelli ${ }^{3,4}$ \\ ${ }^{1}$ Radiology Unit, Hospices Civils de Lyon, Centre Hospitalo-Universitaire Lyon Sud, 69495 Pierre Bénite cedex, France. \\ 2 Inserm UMR 1052, Groupe de Radiobiologie, Centre de Recherche en Cancérologie de Lyon, 28 Rue Laennec, 69008 Lyon, France. \\ ${ }^{3}$ Unit of Radiology, IRCCS Policlinico San Donato, San Donato Milanese, Milan, Italy. \\ ${ }^{4}$ Department of Biomedical Sciences for Health, Università degli Studi di Milano, Milan, Italy.
}

Received: 4 July 2017 / Accepted: 2 October 2017

\begin{abstract}
The aim of this review paper is to clarify the radio-induced breast cancer (BC) risk in BRCA mutation carriers with a combined epidemiological and radiobiological comprehensive approach. A systematic literature search on PubMed (MEDLINE) and EMBASE was performed for articles published from January 1st, 2000 to June 15th, 2017 using dedicated key words as subject headings (MeSH) in three domains of research and evaluation: risk modeling, cohort-studies and ex-vivo radiation biology with epithelial non-tumoral human breast cells. Only eleven articles that meet the selection criteria could be retrieved. These articles are analyzed and discussed. This review, which includes all types of radiological breast exposures, shows an association between $\mathrm{BC}$ risk and low cumulative X-ray doses before age 30 . There is no consistent data in this literature regarding the risk of $\mathrm{BC}$ from radiological exposure after age 30 . Biological data point out strong indicators of radiation-induced genomic instability linked with carcinogenesis pathways. We conclude that the risk of radio-induced BC in BRCA mutation carriers depends on age at exposure and that repeated X-ray breast exposures such as mammography should be used very cautiously in these mutated patients.
\end{abstract}

Keywords: breast cancer / radiation risk / X-ray exposure / BRCA1/2 / breast cancer screening / mammography

\section{Introduction}

BRCA genes have a tumor-suppressing function determining a high risk of breast cancer (BC) in heterozygous mutation carriers (Antoniou et al., 2003). BRCA proteins are critically involved in response to DNA damage, which includes both signaling and repair of DNA breaks. Since cancer process includes a progressive acquisition of persistent DNA damage, the disruption in the DNA damage response related to the BRCA1 or BRCA2 mutated status is involved in $\mathrm{BC}$ development in the carriers. Ionizing radiation, including $\mathrm{X}$-ray radiation, is a well-identified clastogenic factor (DNA observations) and subsequently a well-identified carcinogenic factor (epidemiology observations), notably for the breast considered as one of the most radio-susceptible organ of the body (Ronckers et al., 2005). Because of the high risk of BC, a bilateral mastectomy or an annual screening with imaging modalities is recommended in some countries in BRCA

\footnotetext{
*Corresponding author: catherine.colin01@chu-lyon.fr
}

mutation carriers as early as age 25-35 (Saslow et al., 2007; Sardanelli et al., 2010), mainly including magnetic resonance imaging (MRI) and mammography. These systematic and repeated breast X-ray exposures in women pave the way to controversy in terms of radiation-induced BC risk. In 2010, a meta-analysis conducted by Jansen-van der Weide et al. revealed that low-dose radiation exposures were significantly associated with a higher radiation-induced $\mathrm{BC}$ risk among women with a familial or genetic predisposition (Jansen-van der Weide et al., 2010). They recommended a careful approach by means of reducing repeated exposure, avoidance of exposure at a young age and use of non-ionizing screening techniques.

The goal of this systematic literature review is to clarify this issue with a comprehensive and current approach. Hence, three pathways investigating the radiation-induced $\mathrm{BC}$ risk are considered: risk modeling, cohort-studies data and ex-vivo radiobiological researches. This approach is in line with current international collaborative projects such as EpiRadBio (http://www.epiradbio.eu) and is in the scope of radiological protection new challenges (Bourguignon et al., 2017). 


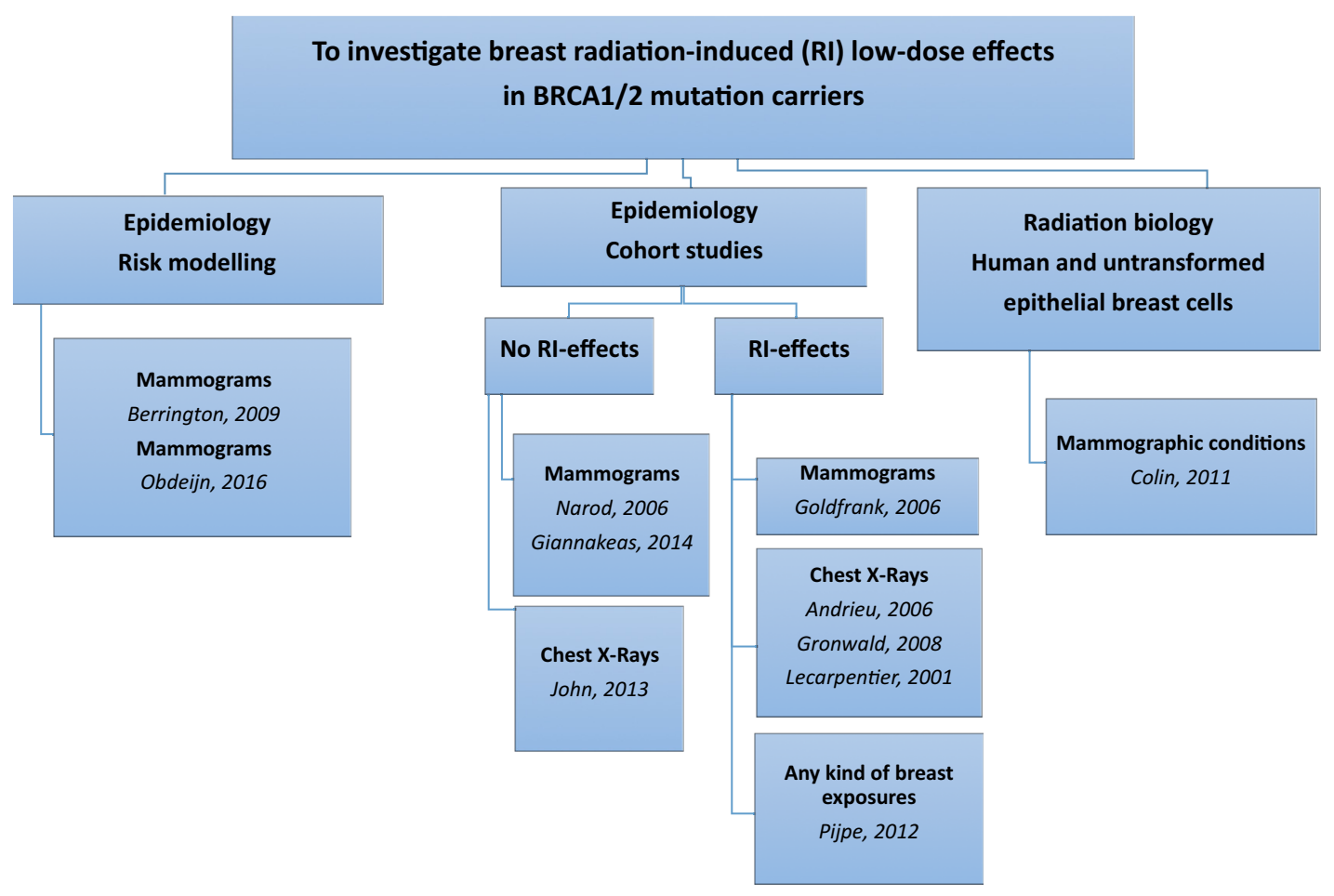

Fig. 1. The holistic approach to assess breast low-dose effects in BRCA1/2 mutation carriers: risk modeling, cohort and dedicated radiobiological studies.

\section{Literature review}

\subsection{Literature search strategy}

A systematic literature search was performed on PubMed (MEDLINE) and EMBASE. The following keywords were used: BRCA1, BRCA2, breast, breast cancer, radiation risk, radiation effects, mammography/adverse effects, mutation/ radiation effects, DNA damage, low-dose radiation, DNA double-strand break (DSB), DNA repair, and breast epithelial cells. These terms were used as subject headings (MeSH) in PubMed or Emtree in EMBASE. The search was restricted to original articles published in English over the period from January 1 st, 2000 to June 15 th, 2017. The primary search was completed by using specific combinations of key words (Appendix A). Studies, which explored radiotherapy effects, were excluded because the issue of radiation-induced $\mathrm{BC}$ after high doses was not in the scope of this review. Then, the following criteria were applied: modeling studies were eligible when evaluating the radiation induced risk in BRCA1/2 mutation carriers; clinical case-control or cohort studies were included if estimating the BC risk in BRCA mutation carriers who underwent mammography or chest $\mathrm{X}$-ray (CXR) or any kind of radiological breast exposures. Computer simulation models comparing radiological screening strategies such as Lowry and colleagues' article (Lowry et al., 2012) are not in the scope of this review dedicated to radiation-induced $\mathrm{BC}$ effects.

Other primary combinations of dedicated key words were performed for radiobiological researches (Appendix B). Biological studies were eligible when using radiological Xray type exposures and when including data from breast cells from BRCA mutation carriers compared with data from cells from women with no identified genetic risk. Since radiation induced carcinogenic effects are strongly dependent on biological species and cellular transformation, only low-dose effect articles using untransformed and non-tumoral human epithelial breast cells were searched.

\subsection{Eligibility and data analysis}

Two readers screened titles and abstracts for eligibility. The full texts of eligible articles were obtained and read in full. References were also cross-checked for further eligibility. After applying this search strategy with strict selection criteria, only eleven articles of the 282 (epidemiology) and 26 (radiobiology) originally identified were retained: there were two on risk projection, eight on epidemiology, and one in radiation biology (Fig. 1).

The same two readers extracted relevant data of the selected articles with consensus reading.

Analyses were put in perspective with the discussion taking into account the three mentioned fields of investigation.

To consider the analysis of the epidemiological studies of this review, we briefly remind the risk models to estimate radiation-induced $\mathrm{BC}$ in the general population, i.e., non BRCA carriers. Female BC risk is one of the most-studied cancer risks after exposure to ionizing radiation and it is well known that exposures increase the risk for $\mathrm{BC}$ occurrence in the general population (Preston et al., 2002). Radiationassociated $\mathrm{BCs}$ occur at ages when spontaneous $\mathrm{BC}$ are seen. There are variations in excess $\mathrm{BC}$ risk estimations with age at exposure, attained age, and time since exposures.

Two dose response models were developed by Preston et al. based on large pooled analyses of eight cohort studies: the 
excess absolute risk (EAR model) and the excess relative risk model (ERR) (Preston et al., 2002). The EAR model assumes that the risk caused by the exposure is independent of the baseline risk and is proportional to the dose. For the EAR model, risk from the exposure is added to the baseline risk (additive model). The ERR model assumes that the risk caused by the exposure is proportional to the baseline risk as well as to the dose. For the ERR model, the risk from exposure is a product of the baseline risk and the dose (multiplicative model). Published results generally indicate that $\mathrm{BC}$ risk in a given population can be described in terms of simple ERR models with a linear dose-response function that tends to decrease with increasing age at exposure (Preston et al., 2002). A question is of how to apply risk estimates derived from one population to another population that may have very different baseline rates. This issue is often discussed in terms of whether the ERR or the EAR is to be preferred when using risk estimates from one population to estimate the effects of an exposure that would be seen in another population.

Hence, ERR and EAR need to be considered for BC risk evaluation.

\section{Modeling $B C$ risk from mammography in BRCA mutation carriers}

Two articles on risk modeling were analyzed: Berrington de Gonzalez et al (Berrington de Gonzalez et al., 2009) and Obdeijn et al. (Obdeijn et al., 2016).

In 2009, Berrington de Gonzales et al. published an estimation of the lifetime risk for radiation-induced $\mathrm{BC}$ in BRCA mutation carriers from five consecutive annual mammographic screening in three age ranges: 25-29, 30-34 and 35-39 years (Berrington de Gonzalez et al., 2009). The results suggest that there would be no benefit on mortality reduction from five annual mammographic screening of $\mathrm{BC}$ before age 35 .

Considering the high-dose-rate X-rays of screening mammograms they used an ERR model based on a pooled analysis of two of the eight cohorts of the study of Preston (Preston et al., 2002) on radiation effects on BC risk: women exposed to multiple fluoroscopies and children whose breasts were exposed during thymic irradiation. Hence, Berrington de Gonzales et al. considered that the assumption underlying the ERR model is that the magnitude of the radiation-induced risk is proportional to the baseline cancer rate of these two-pooled cohorts. Estimates of the baseline $\mathrm{BC}$ incidence rates for BRCA1/2 mutation carriers were taken from a pooled analysis of 22 studies (Antoniou et al., 2003). A mean radiation dose per two-view mammogram was estimated to be $3.85 \mathrm{mGy}$. Considering the publication of Preston (Preston et al., 2002), Berrington and colleagues assumed that the risk increased linearly with dose. Some major points need to be discussed in this study. First, if the dose-response curve at low-doses $(<100 \mathrm{mGy})$ was not linear but curved either upward or downward, this would result in lower or higher risk estimates for mammograms, respectively. Second, Berrington and colleagues estimated the $\mathrm{BC}$ risk from only five mammograms. Indeed, the study was not designed to simulate the effects of 10 to 20 or more annual mammograms, when starting at age 25 , 30 or 35 , as it is commonly recommended by international guidelines (Saslow et al., 2007; Sardanelli et al., 2010). As a consequence, the cumulative effect of long-term annual mammography in these high-risk women was not evaluated. Third, the benefit/risk balance of screening mammography was expressed in terms of mortality and depended on the assumptions regarding the mortality reduction from mammogram screening. Berrington and colleagues calculated the mortality reduction from screening that would be required to outweigh the radiation risk. To estimate this mortality risk/ benefit ratio from screening, they assumed that mammographic screening efficacy for younger high-risk mutation carriers was $15-25 \%$. However, these percentages were derived from randomized controlled trials for average-risk women aged 40 49 years and have never been calculated for BRCA mutation carriers. Thus, this assumption cannot be appropriate for highrisk women or BRCA mutation carriers because BC occurs early in life and screening has to start early. Moreover, mammographic sensitivity for detecting $\mathrm{BC}$ is low due to two reasons: a high probability of an apparently benign $\mathrm{BC}$ appearance in these women and a higher breast density (Sardanelli and Podo, 2007).

The impact of a family history of BC - without BRCA identification - on the radiation-induced $\mathrm{BC}$ risk was previously suggested in modelled risks by Berrington de Gonzalez et al. (Berrington de Gonzalez and Reeves, 2005). The underlying $\mathrm{BC}$ rate was about 2-3 times higher in women with one or two first-degree relatives affected in premenopausal age than in the general population. Consequently, these women were estimated to have a cumulative excess risk of radiation-induced BC mortality approximately 2-3 times higher. In this paper (Berrington de Gonzalez and Reeves, 2005), the relative risk (RR) of BC associated to the family history was translated as the rate of radiation-induced risk increases. Based on this specific translation and modeling from this paper, and taking into account the increased $\mathrm{RR}$ for $\mathrm{BC}$ in BRCA mutations carriers (Tab. 1), the cumulative excess risk of radiation-induced $\mathrm{BC}$ mortality can reach a very high level compared to the general population risk: $14-33$ fold in BRCA1 mutation carriers and 10-20 fold in BRCA2 mutation carriers (Antoniou et al., 2003).

Obdeijn and colleagues (Obdeijn et al., 2016) suggested that postponing screening mammography until age 40 would reduce radiation-induced $\mathrm{BC}$ deaths by $50 \%$ in BRCA1 mutation carriers. The EAR as well as the ERR model were used to explore $\mathrm{BC}$ risks due to a decade of annual mammography in BRCA1 mutation carriers. To analyze the risk, they calculated the mean glandular dose due to a two-view mammogram performed with a digital mammography unit in BRCA1 and BRCA2 mutation carriers and first-degree relatives aged 30 39 years who had mammography during a 1-year period. The mean glandular dose of a two-view mammogram was $4.4 \mathrm{mGy}$ (range 1.7-7.1 mGy). To demonstrate the variation of the risk with the mean dose they also estimated the risk for $1.7 \mathrm{mGy}$ and $7.1 \mathrm{mGy}$ and they demonstrated the consequent variations by using the EAR or the EER model (Tab. 2). Mammographic screening exposures would induce 28 or $208 \mathrm{BC}$ deaths using the EAR and the ERR model respectively per 10,000 women screened. Postponing mammographic screening until age 40 would reduce these numbers by $50 \%$ to 13 or $103 \mathrm{BC}$ deaths respectively according to the EAR and the ERR models per 10,000 women screened. 
Table 1. Relative risk (RR) of breast cancer (95\% CI) of BRCA $1 / 2$ mutation carriers compared to the average risk of the whole population (from Antoniou et al., 2003).

\begin{tabular}{lll}
\hline Age range & BRCA1-RR & BRCA2-RR \\
\hline $20-29$ & $17(4.2-7.1)$ & $19(4.5-8.1)$ \\
$30-39$ & $33(23-49)$ & $16(9.3-29)$ \\
$40-49$ & $32(24-43)$ & $9.9(6.1-16)$ \\
$50-59$ & $18(11-30)$ & $12(7.4-19)$ \\
$60-69$ & $14(6.3-31)$ & $11(6.3-20)$ \\
\hline
\end{tabular}

Table 2. Number of breast cancer deaths due to mammographic screening per 100,000 BRCA1 mutations carriers with annual 2-view mammography from age 30-60 and biennial mammography from age $60-74$ as a function of the mean dose per breast and of the excess risk model (from Obdeijn et al., 2016).

\begin{tabular}{lll}
\hline & Mean dose 1.7 mGy & Mean dose 7.1 mGy \\
\hline EAR model & 11 & 45 \\
ERR model & 80 & 337 \\
\hline
\end{tabular}

In conclusion, the two risk evaluation studies of Berrington de Gonzales et al. and of Obdeijn et al. indicate an excess of $\mathrm{BC}$ risk after mammographic exposure of the breast to ionizing radiation.

\section{Cohort studies involving X-ray low-doses and $\mathrm{BC}$ risk}

Our search retrieved eight epidemiological studies focusing on the effects of low-dose X-rays on BC risk in BRCA1 and/or BRCA2 mutation carriers (Andrieu et al., 2006; Goldfrank et al., 2006; Narod et al., 2006; Gronwald et al., 2008; Lecarpentier et al., 2011; Pijpe et al., 2012; John et al., 2013; Giannakeas et al., 2014). The approaches of these studies are heterogeneous and results are conflicting (Fig. 1 and Tab. 3).

Seven of eight studies assessed the effects of only one radiological modality, i.e., mammography $(n=3)$ or CXR $(n=4)$. Two studies evaluating exposure to only mammography found no association with BC risk (Narod et al., 2006; Giannakeas et al., 2014) while one study showed a modest link for BRCA1 carriers (adjusted odds ratio 1.08; $P=0.03$ ) (Goldfrank et al., 2006). Three of the four studies investigating the effects of only CXR supported an association between early X-ray exposure and $\mathrm{BC}$ risk. By comparing exposed and non-exposed BRCA1/2 mutation carriers, one study reported an increased $\mathrm{BC}$ risk related to any reported CXR exposure of women at age 40 or younger (Andrieu et al., 2006).

All studies included self-reported procedures. Radiological dose level per exam was estimated and standardized in two studies only (Andrieu et al., 2006; Pijpe et al., 2012). No study reported an individual prospective dosimetry, which should have required the implication of medical physicists for any individual radiological exposure. Estimated doses to the breast from radiological exams varied according to time period and countries. These variations were taken into account in two studies (Andrieu et al., 2006; Pijpe et al., 2012).
Studies considering only one radiological examination type, mammogram or CXR, are of limited interest (Tab. 3). They neglected all the other radiological breast exposures. In addition, small numbers of women were reported (Goldfrank et al., 2006; Gronwald et al., 2008; Lecarpentier et al., 2011; John et al., 2013).

Narod and colleagues (Narod et al., 2006) limited their analyses to age at first mammography on the basis of the fact that early mammography is a good indicator of a woman initiating early breast screening. However, a young woman could have mammography only for the assessment of a suspicious mass or other clinical symptoms, and thus this first procedure could not be an indicator of long-term screening behavior. This can potentially constitute a significant bias to stratify the risk linked to the mammogram cumulative dose per woman. Another main bias was pointed out in terms of methodology used to stratify the risk. Whereas the usual recommendation for high-risk women was an annual mammography, for the control group the mean frequency of screening since the date of the first mammography was less than this recommendation - one mammography procedure every 19 months or about 13 mammography procedures over a surveillance period of 20 years.

Giannakeas et al. (2014) showed some major biases to be considered. The hazard ratio was estimated for the development of invasive $\mathrm{BC}$, conditioned by the number of prior mammograms and by the age of the first one. Unfortunately, results were not expressed in terms of dose/effect relation. The cutoff was no mammogram versus one or more mammograms at baseline to define the two populations. The cumulative breast dose per woman remained unknown with no gradient of exposure in mGy explored, whereas stratifying cumulative doses is an imperative condition to explore a potential association between exposure and $\mathrm{BC}$ risk.

The GENE-RAD-RISK study was the only that considered any kind of radiological breast exposure involving chest or shoulders, and dosimetry variations according to time period or countries (Pijpe et al., 2012). The overall results revealed an increased risk of $\mathrm{BC}$ for very low doses. In the cumulative dose category of $6.6 \mathrm{mGy}$ or more per breast, a threefold risk was observed (hazard ratio 3.16, 95\% confidence interval [CI] 1.19-8.36) before age 20. Almost a fourfold $\mathrm{BC}$ risk for an estimated cumulative breast dose equal to or greater than $17 \mathrm{mGy}$ (hazard ratio $3.84,95 \% \mathrm{CI}$ 1.67-8.79) was observed before age 30 at exposure with a dose-response pattern. It was a large European retrospective cohort study in 1993 BRCA mutation carriers. Considering the entire cohort and sometimes several types of exposure per woman, $46 \%(n=919)$ of BRCA mutation carriers reported to have had a radiography somehow involving the breasts, $33 \%$ $(n=649)$ at least a mammogram or more, and $14 \%(n=280)$ a fluoroscopy. Only a small number of BRCA mutation carriers were exposed to computed tomography $1.5 \%(n=29)$ and/or other types of medical radiation exposure such as thyroid scintigraphy $2.7 \%(n=53)$. No association with $\mathrm{BC}$ risk was observed for exposures received after age 30 . However, the follow-up was not long enough to detect a potential association for women exposed at ages 30-40. A potential risk of $\mathrm{BC}$ for exposures after age 30 must be a real concern taking into account the large consensus about the following points on radiation-induced $\mathrm{BCs}$ in the general population 


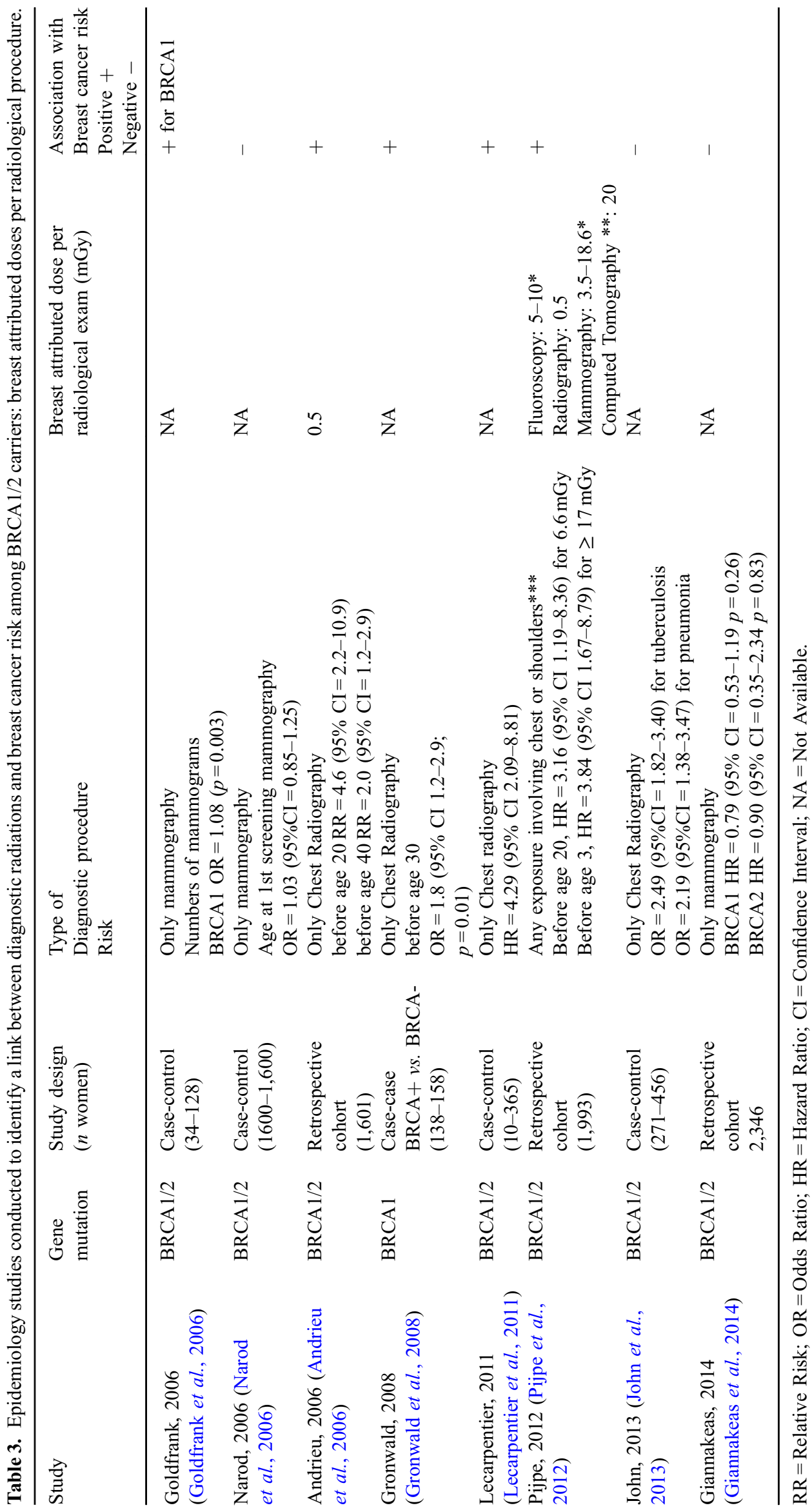


(Mole, 1978; Preston et al., 2002; Ronckers et al., 2005): the risk heavily depends on dose, age at exposure (highly before 20 and proved up to 40); the excess risk appears from about 30 ; the minimum latent period is $10-15$ years after the initial exposure; the excess risk remains up to 50 years after exposure.

Epidemiological cohort data allowed highlighting much lower cumulative dose rates linked to $\mathrm{BC}$ risk in BRCA $1 / 2$ mutation carriers by comparison to the adults of the general population. When evaluating low-dose effects from cohort data in the general population, we face two fundamental limitations. First, the direct estimate is impossible because too large cohorts up to hundreds of thousands of women with a lifelong follow-up are required (Brenner et al., 2003). Second, the exact low-dose response curve remains unknown (Land, 1980). However, important cohort studies demonstrated a link between radiological breast exposures and $\mathrm{BC}$ risk. In adult women exposed to fluoroscopy in the United States and Canada, estimated cumulative doses of $900 \mathrm{mGy}$ were necessary to reveal an association with radiation-induced BCs (Howe and McLaughlin, 1996). But there are major limitations in these fluoroscopic studies regarding our lowdose effect purpose. One of them is related to the "nonexposed" control population defined according to cumulative doses less or equal to $100 \mathrm{mGy}$. So, only the comparison between $\leq 100 \mathrm{mGy}$ and higher doses was explored. The second cohort is related to the general population exposed to multiple radiographic examinations for scoliosis during childhood and adolescence. A level of cumulative estimated dose to the breast of about $100 \mathrm{mGy}$ was associated with a significantly increased $\mathrm{BC}$ rate in adult women (Hoffman et al., 1989; Doody et al., 2000). The ERR per Gy was 5.4\% (95\% CI 1.2-14.1\%) (Doody et al., 2000). Considering an excess risk of BC, standardized incidence ratio was $1.82(90 \%$ CI 1.0-3.0) for the second study (Hoffman et al., 1989).

In conclusion, some cohort studies of BRCA1/2 mutation carriers show an association of $\mathrm{BC}$ risk with very low-dose exposures in comparison with the general population.

\section{Radiobiological study using non-tumoral breast epithelial cells}

We retrieved only one study using non-tumoral and untransformed breast epithelial cells to compare BRCA mutation carriers and high family risk women and those with no family identified risk of breast or ovarian cancer (Colin et al., 2011a). This study highlights radio-induced DNA damage in breast epithelial cells with mammographic lowdose exposure and reveals effects linked to the familial history/ genetic status.

DNA damages were assessed in epithelial cells from breast biopsies performed under ultrasound guidance. A total amount of 250,000 epithelial cells were explored ex-vivo from two categories of women enrolled according to their genetic status or family history of breast/ovarian cancer: women with no identified familial risk and mutated carriers BRCA1, BRCA2 or PTEN and/or at high familial BC risk $(>20 \%-25 \%$ lifetime familial BC risk). Only 4 women were BRCA $1 / 2$ mutation carriers, three of them with a history of contralateral breast radiation therapy. Using an immunofluorescence assay $(\gamma \mathrm{H} 2 \mathrm{AX})$, DNA double strand breaks (DSBs) were quantified before and after X-ray exposure of the human breast cells in the exact conditions of mammography. At this level of dose, the observed increase of $\gamma \mathrm{H} 2 \mathrm{AX}$ foci revealed a lack of DSB recognition and repair. This finding is not new since several studies revealed the existence of radiation-induced DSB and a lack of DNA repair at low-dose (Lobrich et al., 2005; Geisel et al., 2008; Kuefner et al., 2009, 2010) and for doses as low as $1 \mathrm{mGy}$ in non-tumoral and untransformed human cells (Rothkamm and Lobrich, 2003; Grudzenski et al., 2010).

But the study of Colin et al. (2011a) deserves a particular attention because two main specific low-dose effects were observed in addition to the radiation induced DNA-DSBs:

A supra-additive LOw and Repeated Dose (LORD) effect was pointed out and found to be exacerbated in high-risk women (Colin et al., 2011a). Low and repeated doses simulating a 2 -view screening mammogram $(2+2 \mathrm{mGy})$ at 3 minutes interval provided significantly more DSBs than $4 \mathrm{mGy}$, whatever the risk status of the women (Colin and Foray, 2012). Such a supra-additive effect of low-dose X-ray exposure repetition must be carefully considered. According to the current international recommendations in radiation protection, the biological effects of two repeated doses are considered to be equivalent to the sum of the effects due to each dose taken separately. Furthermore, the LORD effect was shown to be strongly dependent on the time interval separating the two doses. If this time is not long enough to allow a complete DSB repair, the second dose may increase the severity of induced DNA damage (Colin et al., 2011a; Viau et al., 2016).

On another hand, repetition of low-doses exposures has been shown to disrupt the breast tissue microenvironment with fibroblast modifications, inducing dysregulated cell-cycle and death pathways of epithelial cells, and increased cytokines and growth factors (Tsai et al., 2005). Thus, in addition to the effects on epithelial cells, radiation-induced effects on the breast stroma after dose repetition are involved in carcinogenesis. The second observed effect called Low-dose ADditional and Induced DSB (LADI) effect is the systematic increase of $\gamma \mathrm{H} 2 \mathrm{AX}$ foci rate per cell from 10 minutes to 24 hours after mammogram exposure (Colin et al., 2011a). The LADI effect is more pronounced in high-risk women. This observation suggests a lack of control in the genome maintenance associated with a hyper-recombination phenomenon. Besides non-homologous end-joining (NHEJ) which consists in ligating the two broken ends of DNA-DSBs, the second major DSB repair pathway is named homologous recombination and consists in cutting some DNA sequences and inserting them in the radiation-induced gaps like a patch system. A lack of control in homologous recombination called hyperrecombination is a well-identified cause of misrepaired DSB, genomic instability and cellular transformation (Gaillard et al., 2015). The highest spontaneous DSB per cell before any ex-vivo radiation exposure in cells extracted from high-risk women in the study of Colin et al. is also an indicator of a hyper-recombination phenomenon. These highly damaged cells can result from a lack of genome maintenance.

There are limitations in the Colin et al. study (Colin et al., 2011a) related to the drawing conclusion on cancer induction from radiobiological studies due to the investigations only 
carried out at the cellular level. Favorable or worsening controls from the immune system, tissue, organ or systemwide mechanisms could not be considered. Notably, the concept of the tumor microenvironment recognizes that the interplay between cancer cells and stromal cells is a crucial determinant of cancer growth (Barcellos-Hoff et al., 2013). In addition to causing gene mutations in epithelial cells protracted low-dose ionizing radiation can contribute to breast carcinogenesis by perturbing human mammary stromal fibroblasts and tissue microenvironment that lead to dysregulated cell-cell and cell-matrix interactions (Tsai et al., 2005).

In conclusion, the abnormal DNA damage level and response observed before and after very low dose exposures (mammography) of epithelial mammary cells of BRCA mutated and high familial risk women may be considered as a strong indicator of the oncogenic risk associated with any type of exposure to ionizing radiation in these carriers.

\section{Radiobiological concern about mammography screening in high-risk women}

Taking into account the genotoxicity of ionizing radiation at doses as low as $1 \mathrm{mGy}$ and the clear evidence that the transformation of a cell into a cancer cell requires the acquisition of successive hallmarks resulting from a poor combination of misrepaired DNA insults (Hanahan and Weinberg, 2011), the alteration of the DNA damage response is at the core of radiation-induced oncogenesis, especially in women carrying a BRCA mutation.

In addition, the contribution of the ATM protein kinase to cell survival after exposure to ionizing radiation and to cancer avoidance has been pointed out (Lobrich and Jeggo, 2005). The ATM protein kinase is a central component of a signal transduction process that responds to DNA DSB by contributing to the phosphorylation of the $\mathrm{H} 2 \mathrm{AX}$ histone variant $(\gamma \mathrm{H} 2 \mathrm{AX})$. Recently, a molecular explanation of the hyper-recombination and hypersensitivity to low-dose exposure was proposed (Bodgi and Foray, 2016): the delay of translocation of ATM from the cytoplasm to the nucleus. In essence, the ATM protein kinase, a major actor of the response to radiation, is mainly localized in the cytoplasm as a dimeric and inactive form. The cytoplasmic ATM forms become monomeric after exposure to radiation and migrate to the nucleus where they participate to the DSB recognition and repair. Any delay in ATM nucleo-shuttling is responsible for a lack of DSB recognition, with consequences. In the case of low-doses ionizing radiation the amount of monomeric ATM forms can be too low to recognize the radiation-induced DSB. For some gene mutations and some individual status, this specific low-dose phenomenon can be particularly exacerbated: low-dose can induce unrepaired DSB (then participating to cell lethality) or misrepaired DNA (then participating to carcinogenesis) (Bodgi and Foray, 2016). Numerous tumor suppressor proteins interact with ATM in cytoplasm, notably BRCA1, BRCA2 and p53, all involved in the response to radiation. Besides, there is previous evidence that BRCA1 and BRCA2 are required for genome surveillance and repair of several DNA damage types, notably DSB (Venkitaraman, 2002; Powell and Kachnic, 2003). Hence, from the mechanistic model described above (Bodgi and Foray, 2016) mutations in tumor suppressor proteins may lead to overexpression of non-functional proteins in cytoplasm that prevent a rapid ATM nucleo-shuttling induced by radiation. The hypersensitivity to low-dose exposure can therefore be particularly observed in cells from high family risk women, mutated or not (Colin et al., 2011b). Cumulative data are currently in favor of an individual response to medical exposures (Foray et al., 2012). Slonina et al. firstly revealed that a low-dose hyper-susceptibility could be an individual characteristic (Slonina et al., 2006, 2007). These authors highlighted more X-ray radiation-induced DNA damages in human cells (fibroblasts and keratinocytes) from some patients at low-dose.

Focusing on screening mammography protocols, this raises specific questions about effects of low-doses, repetition of doses within a few minutes ( 2 systematic mammogram view per breast, cranio-caudal and medio-lateral oblique) or individual factors linked to genetic status and DNA repair capacity.

When evaluating the risk-benefit ratio of mammography and MRI for breast screening in high-risk population, an appropriate estimation of the risk of radiation-induced $\mathrm{BC}$ from low-dose X-ray exposure in increased $\mathrm{BC}$ risk women is needed, especially for BRCA mutation carriers.

The paradigm (MRI as an adjunct to mammography) cannot be simply applied, at least to BRCA mutation carriers. The annual basic mammographic protocol (2 views per breast) in high family risk women should be rethought (Colin and Foray, 2012). Taking into account MRI advances in BC screening, notably for ductal carcinoma in situ, one single medio-lateral oblique view can be proposed with MRI to detect microcalcifications (Colin and Foray, 2012; Colin et al., 2012). The exposure-dose would be at least halved and the radiationinduced effects probably even more reduced considering potential LORD effect and the hypercombination phenomenon in DNA damage responses in such women. If MRI is performed, a guideline suggested the possibility to completely avoid mammography up to 35 years of age (Sardanelli et al., 2010). Interestingly, a recent individual-patient-data metaanalysis pointed out that the relative contribution of mammography for $\mathrm{BC}$ screening (i.e., cases of mammography-only detection, with false negative MRI) in BRCA mutation carriers is relatively low particularly for BRCA1 mutation carriers (Phi et al., 2016). Finally, it has been suggested to perform MRI associated with only one systematic mammographic view per breast to detect calcifications in order to limit breast X-ray low doses (Colin et al., 2012).

\section{Conclusion}

In conclusion, while cumulative evidence for abnormal individual responses to ionizing radiation were reported (Foray et al., 2012, 2016), this review alerts specifically on the $\mathrm{BC}$ risk due to ionizing radiation exposure in BRCA mutation carriers.

Modeling notifies the risk linked to mammogram screening. Moreover, we alert on the main variations of risk linked to the choice of the mean dose per breast and the choice of the excess risk model (EAR or ERR). A strong association 
with cancer is highlighted for very low doses before age 30 with no dedicated data investigating exposure effects after age 30. At a cellular level, significant radiation-induced genomic instability, which is reflected by the hyper-recombination phenomenon, has been observed in cells from high familial risk women. Since genomic instability is systematically observed in all cancer-prone diseases and result from the lack of control of the recombination DNA repair pathways, the radiation-induced genomic instability observed in cells from high familial risk women is a sign of an increased risk of BC. These cellular observations are in agreement with warning epidemiological data where BRCA1/2 mutation carriers BC risk is linked with very low-doses compared to the general population. Because a large prospective cohort of BRCA1/2 mutation carriers recently demonstrated the potential importance of family history and mutation location in risk of $\mathrm{BC}$, we suppose an impact of such parameters on radiation-induced BC risk (Kuchenbaecker et al., 2017). Some gene variants can potentially indicate some radiation-sensitive subgroups of BRCA1/2 mutation carriers.

While BRCA gene mutations were discovered in 19941995 and radiation-induced $\mathrm{BC}$ proved up to age 40 in the general population, we are waiting for consistent cohort data with long follow-up about the consequence of cumulative and repeated ionizing radiation breast exposure after age 30 . In clinical practice, indications for any kind of ionizing radiation exposure involving breast, chest, or shoulders should be systematically and carefully justified in BRCA mutation carriers and other very high familial risk women ( $>20 \%-25 \%$ lifetime familial BC risk with non-identified mutation and untested with first-degree relative with BRCA1 or BRCA2 gene mutation). In addition, because the BRCA mutation screening test is allowed only at adult age, any radiological X-ray examination including the thorax for children of a mutation carrier (woman or man) should be also carefully justified.

\section{Abbreviations}

$\begin{array}{ll}\text { ATM } & \text { Ataxia-telangiectasia mutated } \\ \text { BC } & \text { Breast cancer } \\ \text { CXR } & \text { Chest X-ray } \\ \text { DNA } & \text { Deoxyribonucleic acid } \\ \text { DSB } & \text { double-strand break } \\ \text { LADI } & \text { Low-dose ADditional and dose-induced (effect) } \\ \text { LORD } & \text { LOw and Repeated Dose (effect) } \\ \text { NHEJ } & \text { non-homologous end-joining } \\ \text { ERR } & \text { excess relative risk } \\ \text { EAR } & \text { excess absolute risk } \\ \gamma H 2 A X & \text { H2AX histone } \gamma \text { variant }\end{array}$

\section{Appendix A : Literature search - Epidemiology}

The search was restricted to original articles published in English over the period from January 1st, 2000 to June 15th, 2017. Some combinations completed the primary search: ("genes, brca1"[MeSH Terms] OR ("genes"[All Fields] AND
"brca1"[All Fields]) OR "brca1 genes"[All Fields] OR "brca1"[All Fields]) AND ("genes, brca2"[MeSH Terms] OR ("genes"[All Fields] AND "brca2"[All Fields]) OR "brca2 genes"[All Fields] OR "brca2"[All Fields]) AND ("radiation"[MeSH Terms] OR "radiation"[All Fields] AND ("breast neoplasms"[MeSH Terms] OR ("breast"[All Fields] AND "neoplasms"[All Fields]) OR "breast neoplasms"[All Fields] OR ("breast"[All Fields] AND "cancer"[All Fields]) OR "breast cancer"[All Fields]).

\section{Appendix B : Literature search - Radiobiology}

Other primary combinations were performed for radiobiological researches with dedicated keyword combinations; "dna damage"[MeSH Terms] OR ("dna"[All Fields] AND "damage"[All Fields]) OR "dna damage"[All Fields]) AND ("mammography"[MeSH Terms] OR "mammography"[All Fields] with 26 articles; "dna damage"[MeSH Terms] OR ("dna"[All Fields] AND "damage"[All Fields]) OR "dna damage"[All Fields]) AND ("thorax"[MeSH Terms] OR "thorax"[All Fields] OR "chest"[All Fields]) AND ("diagnostic imaging"[Subheading] OR ("diagnostic"[All Fields] AND "imaging"[All Fields]) OR "diagnostic imaging"[All Fields] OR "x ray"[All Fields] OR "x-rays"[MeSH Terms] OR "x-rays"[All Fields] with 27 articles; "dna damage"[MeSH Terms] OR ("dna"[All Fields] AND "damage"[All Fields]) OR "dna damage"[All Fields]) AND ("tomography, x-ray computed"[MeSH Terms] OR ("tomography"[All Fields] AND "x-ray"[All Fields] AND "computed"[All Fields]) OR "x-ray computed tomography"[All Fields] OR ("computed"[All Fields] AND "tomography"[All Fields]) OR "computed tomography"[All Fields] with 158 articles.

\section{References}

Andrieu N, Easton DF, Chang-Claude J, et al. 2006. Effect of chest $\mathrm{X}$-rays on the risk of breast cancer among BRCA1/2 mutation carriers in the international BRCA1/2 carrier cohort study: a report from the EMBRACE, GENEPSO, GEO-HEBON, and IBCCS Collaborators' Group, J. Clin. Oncol. 24: 3361-3366.

Antoniou A, Pharoah PD, Narod S, et al. 2003. Average risks of breast and ovarian cancer associated with BRCA1 or BRCA2 mutations detected in case Series unselected for family history: a combined analysis of 22 studies, Am. J. Hum. Genet. 72: 1117-1130.

Barcellos-Hoff MH, Lyden D, Wang TC. 2013. The evolution of the cancer niche during multistage carcinogenesis, Nat. Rev. Cancer 13: $511-518$.

Berrington de Gonzalez A, Reeves G. 2005. Mammographic screening before age 50 years in the UK: comparison of the radiation risks with the mortality benefits, Br. J. Cancer 93: 590596.

Berrington de Gonzalez A, Berg CD, Visvanathan K, Robson M. 2009. Estimated risk of radiation-induced breast cancer from mammographic screening for young BRCA mutation carriers, J. Natl. Cancer Inst. 101: 205-209.

Bodgi L, Foray N. 2016. The nucleo-shuttling of the ATM protein as a basis for a novel theory of radiation response: resolution of the linear-quadratic model, Int. J. Radiat. Biol. 92: 117-131.

Bourguignon M, Bérard P, Bertho JM, Farah J, Mercat C. 2017. What's next in Radioprotection? Radioprotection 52: 21-28. 
Brenner DJ, Doll R, Goodhead DT, et al. 2003. Cancer risks attributable to low doses of ionizing radiation: assessing what we really know, Proc. Natl. Acad. Sci. U.S.A 100: 13761-13766.

Colin C, Foray N. 2012. DNA damage induced by mammography in high family risk patients: only one single view in screening, Breast 21: 409-410.

Colin C, Devic C, Noel A, et al. 2011a. DNA double-strand breaks induced by mammographic screening procedures in human mammary epithelial cells, Int. J. Radiat. Biol. 87: 1103-1112.

Colin C, Granzotto A, Devic C, Viau M, Maalouf M, Vogin G, Joubert A, Thomas C, Foray N. 2011b. MRE11 and H2AX biomarkers in the response to low-dose exposure: Balance between individual susceptibility to radiosensitivity and to genomic instability, Int. J. Low Radiation 8: 96-106.

Colin C, de Vathaire F, Noel A, et al. 2012. Updated relevance of mammographic screening modalities in women previously treated with chest irradiation for Hodgkin disease, Radiology 265: 669-676.

Doody MM, Lonstein JE, Stovall M, Hacker DG, Luckyanov N, Land CE. 2000. Breast cancer mortality after diagnostic radiography: findings from the U.S. Scoliosis Cohort Study, Spine (Phila Pa 1976) 25: 2052-2063.

Foray N, Colin C, Bourguignon M. 2012. 100 years of individual radiosensitivity: how we have forgotten the evidence, Radiology 264: 627-631.

Foray N, Bourguignon M, Hamada N. 2016. Individual response to ionizing radiation, Mutat. Res. 770: 369-386.

Gaillard H, Garcia-Muse T, Aguilera A. 2015. Replication stress and cancer, Nat. Rev. Cancer 15: 276-289.

Geisel D, Heverhagen JT, Kalinowski M, Wagner HJ. 2008. DNA double-strand breaks after percutaneous transluminal angioplasty, Radiology 248: 852-859.

Giannakeas V, Lubinski J, Gronwald J, et al. 2014. Mammography screening and the risk of breast cancer in BRCA1 and BRCA2 mutation carriers: a prospective study, Breast Cancer Res. Treat. 147: 113-118.

Goldfrank D, Chuai S, Bernstein JL, et al. 2006. Effect of mammography on breast cancer risk in women with mutations in BRCA1 or BRCA2, Cancer Epidemiol. Biomarkers Prev. 15: 2311-2313.

Gronwald J, Pijpe A, Byrski T, et al. 2008. Early radiation exposures and BRCA1-associated breast cancer in young women from Poland, Breast Cancer Res. Treat. 112: 581-584.

Grudzenski S, Raths A, Conrad S, Rube CE, Lobrich M. 2010. Inducible response required for repair of low-dose radiation damage in human fibroblasts, Proc. Natl. Acad. Sci. U.S.A. 107: 14205-14210.

Hanahan D, Weinberg RA. 2011. Hallmarks of cancer: the next generation, Cell 144: 646-674

Hoffman DA, Lonstein JE, Morin MM, Visscher W, Harris BS, 3rd, Boice JD, Jr. 1989. Breast cancer in women with scoliosis exposed to multiple diagnostic X-rays, J. Natl. Cancer Inst. 81: $1307-1312$.

Howe GR, McLaughlin J. 1996. Breast cancer mortality between 1950 and 1987 after exposure to fractionated moderate-dose-rate ionizing radiation in the Canadian fluoroscopy cohort study and a comparison with breast cancer mortality in the atomic bomb survivors study, Radiat. Res. 145: 694-707.

Jansen-van der Weide MC, Greuter MJ, Jansen L, Oosterwijk JC, Pijnappel RM, de Bock GH. 2010. Exposure to low-dose radiation and the risk of breast cancer among women with a familial or genetic predisposition: a meta-analysis, Eur. Radiol. 20: 25472556 .
John EM, McGuire V, Thomas D, et al. 2013. Diagnostic chest X-rays and breast cancer risk before age 50 years for BRCA1 and BRCA2 mutation carriers, Cancer Epidemiol. Biomarkers Prev. 22: 15471556.

Kuchenbaecker KB, Hopper JL, Barnes DR, et al. 2017. Risks of breast, ovarian, and contralateral breast cancer for BRCA1 and BRCA2 mutation carriers, JAMA 317: 2402-2416.

Kuefner MA, Grudzenski S, Schwab SA, et al. 2009. DNA doublestrand breaks and their repair in blood lymphocytes of patients undergoing angiographic procedures, Invest. Radiol. 44: 440-446.

Kuefner MA, Grudzenski S, Hamann J, et al. 2010. Effect of CT scan protocols on x-ray-induced DNA double-strand breaks in blood lymphocytes of patients undergoing coronary CT angiography, Eur. Radiol. 20: 2917-2924.

Land CE. 1980. Estimating cancer risks from low doses of ionizing radiation, Science 209: 1197-1203.

Lecarpentier J, Nogues C, Mouret-Fourme E, et al. 2011. Variation in breast cancer risk with mutation position, smoking, alcohol, and chest X-ray history, in the French National BRCA1/2 carrier cohort (GENEPSO), Breast Cancer Res. Treat. 130: 927-938.

Lobrich M, Jeggo PA. 2005. The two edges of the ATM sword: cooperation between repair and checkpoint functions, Radiother. Oncol. 76: 112-118.

Lobrich M, Rief N, Kuhne M, et al. 2005. In vivo formation and repair of DNA double-strand breaks after computed tomography examinations, Proc. Natl. Acad. Sci. U.S.A. 102: 8984-8989.

Lowry KP, Lee JM, Kong CY, et al. 2012. Annual screening strategies in BRCA1 and BRCA2 gene mutation carriers: a comparative effectiveness analysis, Cancer 118: 2021-2030.

Mole RH. 1978. The sensitivity of the human breast to cancer induction by ionizing radiation, Br. J. Radiol. 51: 401-405.

Narod SA, Lubinski J, Ghadirian P, et al. 2006. Screening mammography and risk of breast cancer in BRCA1 and BRCA2 mutation carriers: a case-control study, Lancet Oncol. 7: 402-406.

Obdeijn IM, Heijnsdijk EA, Hunink MG, Tilanus-Linthorst MM, de Koning HJ. 2016. Mammographic screening in BRCA1 mutation carriers postponed until age 40: evaluation of benefits, costs and radiation risks using models, Eur. J. Cancer 63: 135-142.

Phi XA, Saadatmand S, De Bock GH, et al. 2016. Contribution of mammography to MRI screening in BRCA mutation carriers by BRCA status and age: individual patient data meta-analysis, $B r . J$. Cancer 114: 631-637.

Pijpe A, Andrieu N, Easton DF, et al. 2012. Exposure to diagnostic radiation and risk of breast cancer among carriers of BRCA $1 / 2$ mutations: retrospective cohort study (GENE-RAD-RISK), $B M J$ 345: e5660.

Powell SN, Kachnic LA. 2003. Roles of BRCA1 and BRCA2 in homologous recombination, DNA replication fidelity and the cellular response to ionizing radiation, Oncogene 22: 5784-5791.

Preston DL, Mattsson A, Holmberg E, Shore R, Hildreth NG, Boice JD, Jr. 2002. Radiation effects on breast cancer risk: a pooled analysis of eight cohorts, Radiat. Res. 158: 220-235.

Ronckers CM, Erdmann CA, Land CE. 2005. Radiation and breast cancer: a review of current evidence, Breast Cancer Res. 7: 21-32.

Rothkamm K, Lobrich M. 2003. Evidence for a lack of DNA doublestrand break repair in human cells exposed to very low x-ray doses, Proc. Natl. Acad. Sci. U.S.A. 100: 5057-5062.

Sardanelli F, Podo F. 2007. Breast MR imaging in women at high-risk of breast cancer. Is something changing in early breast cancer detection? Eur. Radiol. 17: 873-887.

Sardanelli F, Boetes C, Borisch B, et al. 2010. Magnetic resonance imaging of the breast: recommendations from the EUSOMA working group, Eur. J. Cancer 46: 1296-1316. 
Saslow D, Boetes C, Burke W, et al. 2007. American Cancer Society guidelines for breast screening with MRI as an adjunct to mammography, CA Cancer J. Clin. 57: 75-89.

Slonina D, Biesaga B, Urbanski K, Kojs Z, Waligorski M. 2006. Evidence of low-dose hyper-radiosensitivity in normal cells of cervix cancer patients? Radiat. Prot. Dosimetry 122: 282-284.

Slonina D, Biesaga B, Urbanski K, Kojs Z. 2007. Low-dose radiation response of primary keratinocytes and fibroblasts from patients with cervix cancer, Radiat. Res. 167: 251-259.
Tsai KK, Chuang EY, Little JB, Yuan ZM. 2005. Cellular mechanisms for low-dose ionizing radiation-induced perturbation of the breast tissue microenvironment, Cancer Res. 65: 6734-6744.

Venkitaraman AR. 2002. Cancer susceptibility and the functions of BRCA1 and BRCA2, Cell 108: 171-182.

Viau M, Perez AF, Bodgi L, et al. 2016. Repeated radiation dose effect and DNA repair: Importance of the individual factor and the time interval between the doses, Cancer Radiother. 20: $217-225$

Cite this article as: Colin C, Foray N, Di Leo G, Sardanelli F. 2017. Radiation induced breast cancer risk in BRCA mutation carriers from low-dose radiological exposures: a systematic review. Radioprotection 52(4): 231-240 